\title{
Sudden Death of a Child due to Unidentified Chronic Myocarditis: Autopsy Findings
}

\author{
Ilma Kurtagić Pećanin ${ }^{1}$, Fehim Juković ${ }^{1}$, Suzana Matejić ${ }^{2}$ Azra Juković ${ }^{3}$, Selmina Nicević ${ }^{4}$, Ruža Milanović \\ Kaličanin ${ }^{3}$ \\ ${ }^{1}$ General Hospital of Novi Pazar, Department of Pathology and Forensic Medicine, Novi Pazar, Serbia; ${ }^{2}$ University of Priština, \\ Institute of Forensic Medicine, Faculty of Medicine, Kosovska Mitrovica, Serbia; ${ }^{3}$ General Hospital of Novi Pazar, Department \\ of Pediatrics, Novi Pazar, Serbia; ${ }^{4}$ General Hospital of Novi Pazar, Department of Neurology, Novi Pazar
}

Correspondence: kurtagic.ilma@gmail.com; Tel.: + 381628768 586; Fax.: + 38120331151

Received: December 27, 2019; Accepted: February 12, 2020

\begin{abstract}
Objective - In this paper, we present the case of the sudden cardiac death of an apparently healthy 15-year-old adolescent, as well as the autopsy findings, with histopathology. Case report - A 15-year-old boy was found unconscious in his brother's room. The boy had not been diagnosed with any disease and was apparently healthy. Preliminary autopsy findings indicated the predominance of cardiac pathology in relation to his death. All the cardiac cavities were dilated and enlarged. The trabeculae were more pronounced and thicker, with deepened spaces between them. In the anterior wall and almost the entire upper half of the posterior wall of the left ventricle, as well as the interventricular septum, with almost the entire thickness of the wall, the myocardial tissue was densely imbued with fibrous tissue. Microscopic analysis of cardiac sections showed large fields of hypocellular (scarring) fibrosis, as well as smaller fibrosis focuses of moderate cellularity, with fibroblasts, fibrocytes, and lymphocytes present. Toxicological analysis of blood taken from the femoral vein during autopsy was also performed, and the results showed that there were no traces of alcohol or psychoactive substances. Conclusion - Myocarditis is not uncommon in the pediatric population and has a relatively good prognosis, with most cases resulting in complete recovery. Extensive chronic inflammation with severe fibrosis in an apparently healthy boy leading to death makes our case particularly significant.
\end{abstract}

Key Words: Myocarditis • Fatal Outcome • Autopsy • Adolescent • Dilated Cardiomyopathy.

\section{Introduction}

Heart diseases are among the leading causes of sudden and unexpected natural deaths in the population of children and young adults (1). Unlike adults, in whom sudden cardiac death (SCD) is usually a consequence of atherosclerotic coronary artery disease, in children SCD is most likely due to myocarditis, cardiomyopathy or congenital cyanogenic heart defects (1-3). Myocarditis is one of the most common causes of SCD diagnosed after autopsy and microscopic examinations (4). A significant number of myocarditis go unnoticed, due to nonspecific symptomatology or because of subclinical presentations, so the true incidence is unknown. However, thanks to systematic postmortem studies, myocarditis has been shown to be much more common than previously thought (57). Some autopsy studies have shown that myocarditis is the cause of death in up to $36 \%$ of the population of children aged 0 to 18 years, with sudden death being the first manifestation of the disease in more than half $(5,8)$. The etiology of myocarditis is diverse. In the pediatric population, a large percentage of myocardial inflammation is a result of viral infection, acute or chronic. It has been shown that a chronic infection is often associated with dilated cardiomyopathy $(5,9)$. Nevertheless, inflam- 
mation of the cardiac muscle of viral origin in most children will result in complete restitution, while in only a small number it will cause irreversible morphological changes with impaired structural and functional integrity of the cardiac muscle.

In this paper, we present the case of the sudden cardiac death of an apparently healthy 15-year-old adolescent, as well as the autopsy findings, with histopathological tissue analysis.

\section{Case Report}

A 15-year-old boy was found unconscious in his brother's room. The emergency medical services were called, but the boy was already dead. Due to the unclear circumstances in this apparently healthy boy, the doctor asked for a clinical autopsy to determine the cause of death.

An analysis of the available medical records and heteroanamestic data obtained from the parents revealed that the boy had not been diagnosed with any disease and was apparently healthy. He had frequent episodes of coughing, which had been explained by the pediatrician as acute respiratory infections of the upper respiratory tract. Standard blood tests were performed during visits to the doctor's office (complete blood count and estimated sedimentation rate), with results being consistent with acute infection. The last recorded episode of this was more than 6 months prior to death. He was actively involved in dance. He was not taking any psychoactive substances nor alcohol. At the time of death, the boy was at a party and, according to information received from friends, he felt chest pain while dancing, after which he went out to another room, where he was found.

External examination revealed that the body was of average build. Preliminary autopsy findings indicated the predominance of cardiac pathology in relation to his death. Subepicardial hemorrhages were noted, with several foci on the front of the heart and larger ones on the posterior side, as well as a smaller region of subendocardial hemorrhage in the left ventricle. All the cardiac cavities were dilated and enlarged. The trabeculae were more pronounced and thicker, with deepened spaces between them. In the anterior wall and over almost the entire upper half of the posterior wall of the left ventricle, as well as the interventricular septum, and covering almost the entire thickness of the wall, the myocardial tissue was densely imbued with fibrous tissue. The heart muscle in the region of the interventricular septum, in almost its entire length, was pervaded with blood (Fig. 1).

The sections of the lungs showed foci of bleeding, with signs of blood aspiration. The abdominal organs (liver and spleen) were hyperemic and easily enlarged. Macroscopic examination revealed no other morphological changes. During the autopsy, standard tissue samples were taken for further pathohistological analysis, with the number of samples taken from heart tissue being according to the pathohistological protocol for suspected myocarditis, which is why 4 sections from the left ventricle, two from the right ventricle and two from the intraventricular septum were taken.

Microscopic analysis of the cardiac sections showed large fields of hypocellular (scarring) fibrosis, as well as smaller fibrosis focuses of moderate cellularity, with fibroblasts, fibrocytes, and lymphocytes present. Focal lymphocyte infiltration, with signs of acute myocardial damage, was also observed. The finding was consistent with chronic inflammation of the heart muscle in exacerbation (Fig. 2). Fresh focal intraalveolar hemorrhage emphysema was observed in the lungs. The liver tissue sections showed that the port areas were easily expanded, slightly infiltrated by a mixed inflammatory infiltrate, b but without fibrosis. Toxicological analyses of blood taken from the femoral vein during autopsy were also performed, and the results showed that there were no traces of alcohol or psychoactive substances. 

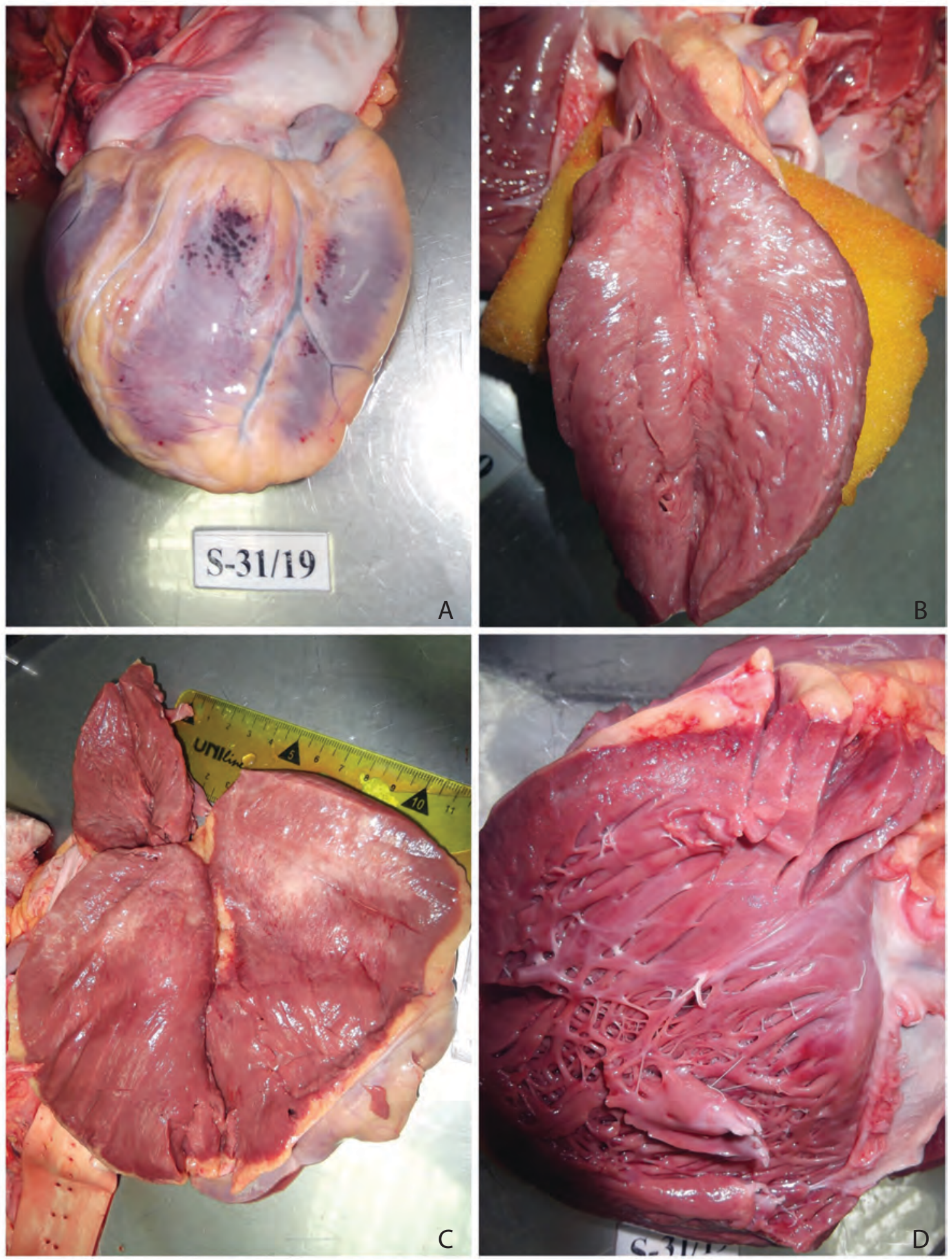

Fig. 1. Macroscopic Appearance of the Heart. A-Subepicardial Hemorrhages; B- Fibrosis in Anterior Wall of Left Chamber; C-Fibrosis in Posterior Wall of Left Chamber; D-Region of Subendocardial Hemorrhage in the Left Ventricle with the Interventricular Septum Pervaded With Blood. 

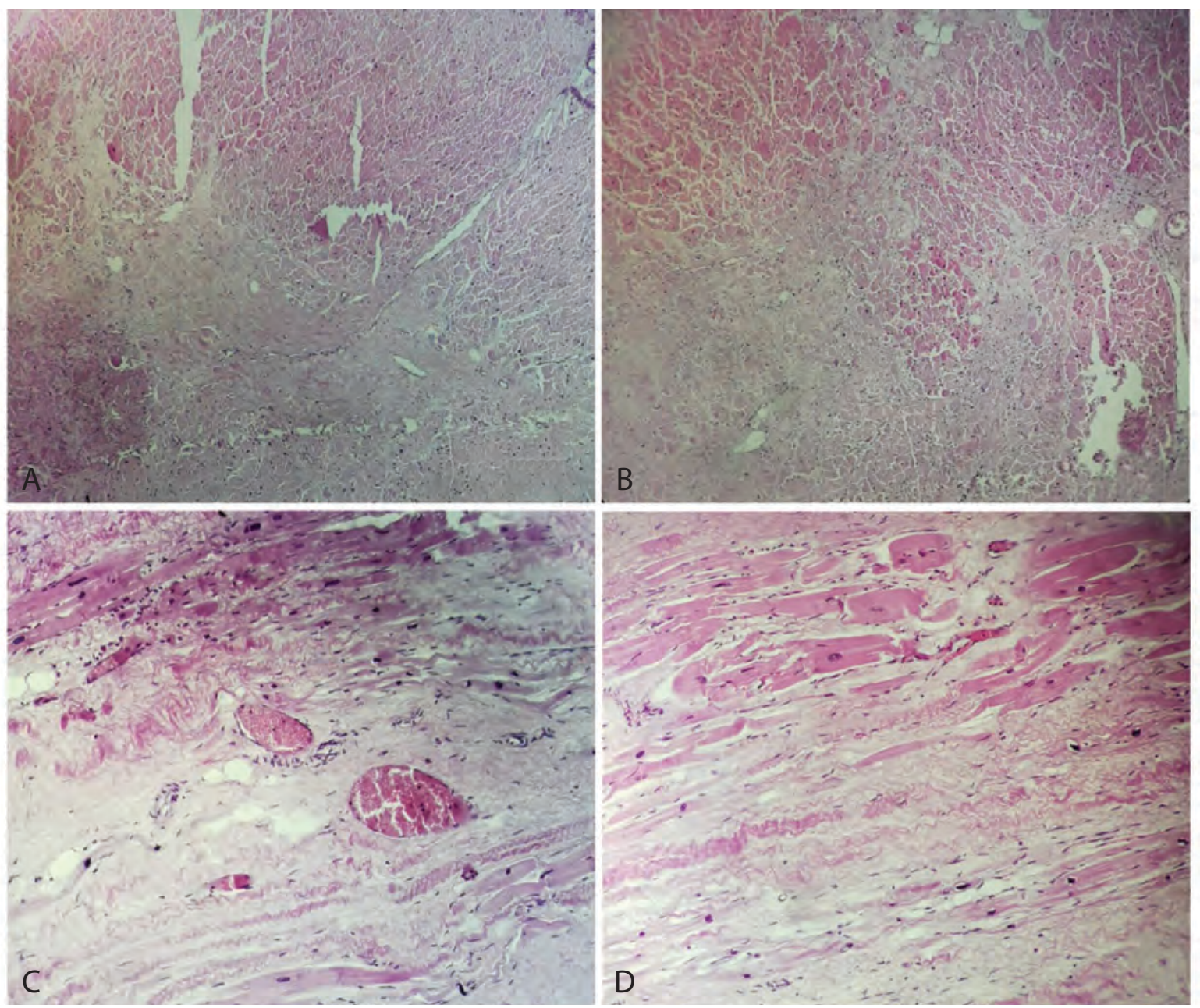

Fig. 2. Microscopic Appearance of the Myocardial Sections. A, B-Fields of Hypocellular Fibrosis with Areas of Viable Myocardium in Sections from the Anterior (A) and Posterior (B) Walls; C, D - Focuses of Fibrosis Moderate Cellularity, with Fibroblasts, Fibrocytes, and Lymphocytes Present.

\section{Discussion}

Unexpected, abrupt and sudden deaths in children and adolescents are very rare, with a range of $1.3-$ $8 \%$ per 100,000 per year. The cause of death in the pediatric population may be of varied origin, including cardiac (3). In the study by Ilina et al. it was shown that as many as $35.9 \%$ cases of sudden death occurred due to myocarditis, and further pathohistological analysis showed that in more than two-thirds of cases inflammation with lymphocytic infiltrate was present (8). In our case, microscopic analysis of samples from the heart showed areas of extensive scarring, fibrosis, with focal lymphocytic infiltration, corresponding to worsening chronic myocarditis, and this confirmed the macroscopic diagnosis made during the autopsy. The drastic myocardial findings observed during the autopsy could not have occurred during a single attack of viral infection, but there were, probably, multiple episodes of infection where each left a mark on the heart muscle. Although serology testing was not performed in this case, due to technical demerits, potential causative agents include Coxsackievirus group B (an enterovirus), Parvovirus B19, Human Herpes Virus 6, as well as Echoviruses (known to cause gastrointestinal infection) and Epstein-Barr 
virus. Macroscopic diagnosis of diffuse cardiac fibrosis, which is caused by chronic myocarditis, is rarely seen during autopsy in young people. Immediately before his death, the boy had no medical conditions or symptoms of heart disease. Particularly important and confusing is the information obtained from the parents that the boy engaged actively and without difficulty in ethno-dance several times a week (he undertook regular intense physical activity), which is unusual given the finding on the heart. Prior to his death, the boy was also subjected to great physical exertion. The likely mechanism of the disorders that led to death can be explained by the onset of fatal arrhythmia in the region of the fibrous altered myocardium, with the development of dilated cardiomyopathy. Acute inflammation of the myocardium may not only damage the cardiomyocytes, but also elements of the conduction system, and cause arrhythmias, while in the chronic phase, myocardial scaring leads to electrical instability, reentry arrhythmias and rhythm disturbances (4).

The prevalence of children with chronic myocarditis who will develop arrhythmias ranges from $10-30 \%$ and later in life they will have high probability of developing dilatative cardiomyopathy (10, 11 ), since myocarditis is one of the most common causes of so-called secondary dilatative cardiomyopathies $(12,13)$. It is reported in the literature that the most common course of events is the occurrence of ventricular tachycardia, with progression to ventricular fibrillation and then asystole. It is even thought that in up to $80 \%$ of cases with sudden cardiac death, the first ECG recording will show ventricular fibrillation (3). The pitfall in our case was that during systematic school examinations the boy never underwent an ECG recording or any other diagnostic procedure to assess cardiac function.

\section{Conclusion}

Myocarditis in infancy is not uncommon and has a relatively good prognosis $(14,15)$, so most will experience complete recovery. Extensive chronic inflammation with severe fibrosis in a seemingly healthy adolescent which led to death is what makes our case special in relation to the available literature.
Authors' Contributions: Conception and design: IKP and FJ; Acquisition, analysis and interpretation of data: FJ, RKM, AJ and SNN; Drafting the article: IKP, FJ and SNN; Revising the article critically for intellectual content: AJ, RKM and SM; Approved final version of the manuscript: FJ and IKP.

Conflict of Interest: The authors declare that they have no conflict of interest.

\section{References}

1. Byard RW. Sudden death in young. 3rd ed. New York: Cambridge University Press; 2010.

2. Bonsignore A, Palmiere C, Buffelli F, Maselli E, Marzullo A, Fraternali Orcioni G, et al. When is myocarditis indeed the cause of death?. Forensic Sci Int. 2018;285:72-6. doi:10.1016/j.forsciint.2018.01.027.

3. Capucci A, editor. Sudden cardiac death. London: Informa Heathcare; 2007.

4. Byard RW. Sudden death in Infancy, Childhood and Adolescence. 2nd ed. New York: Cambridge University Press; 2004.

5. Madea B, editor. Handbook of forensic medicine. Chichester: Wiley Blackwell; 2014.

6. Blauwet LA, Cooper LT. Myocarditis. Prog Cardiovasc Dis. 2010; 52(4): 274-88.

7. Kühl U, Schultheiss HP. Myocarditis in Children. Heart Failure Clin. 2010;6:483-96.

8. Ilina MV, Kepron CA, Taylor GP, Perrin DG, Kantor PF, Somers GR. Undiagnosed Heart Disease Leading to Sudden Unexpected Death in Childhood: A Retrospective Study. Pediatrics. 2011;128:513-20.

9. Edwards BS, Edwards JE. Pathology of sudden cardiac death: An Illustrated Guide. Malden: Wiley-Blackwell; 2006.

10. Huber SA. Viral myocarditis and dilated cardiomyopathy: Etiology and pathogenesis. Curr Pharm. 2016;22:408-26.

11. Fu MY, Wang QW, Xue Y, Xu F, Li CL, An XJ. Relevant researches on chronic viral myocarditis (CVMC) in children, complicated with arrhythmia and thyroid hormone level. Eur Rev Med Pharmacol Sci. 2017;21:3083-7.

12. Haas NA, Kleideiter U. Pediatric cardiology: SymptomsDiagnosis-Treatment. Stuttgart: Thieme; 2011.

13. Keane JF, Lock JE, Fyler DC. Nadas' Pediatric Cardiology. Philadelphia: Elsevier; 2006.

14. Ethan L. Viral myocarditis. The Pediatric Infectious Disease. 2004;23(7):665-6.

15. Dancea AB. Myocarditis in infants and children: a review for the paediatrician. Paediatric and child health. 2001;6(8):543-5. 